\title{
A Case Report of IgG4-related Diseases Involving Mammary Gland, Parotid Gland and Orbit
}

\author{
Ruixia Yang ${ }^{1,2, \#}$, Weijuan Song ${ }^{1,2, \#}$, Ting Cui ${ }^{1,2, \#}$, Qiuxia Ge ${ }^{1,2}$, Li Jiang ${ }^{1,2, *}$, Lujiang Yi $^{1,2, *}$ \\ ${ }^{1}$ Department of Laboratory Medicine, The First Affiliated Hospital of Nanjing Medical University, Nanjing, P. R. China \\ ${ }^{2}$ National Key Clinical Department of Laboratory Medicine, Nanjing, P. R. China. \\ "These authors contributed equally to this work. \\ *Corresponding author: joy232295@163.com;158126189@qq.com
}

Received March 10, 2019; Revised April 17, 2019; Accepted April 26, 2019

\begin{abstract}
Immunoglobulin G4-related disease (IgG4-RD) is a chronic progressive autoimmune disease. Mammary gland, parotid gland and orbit involvement in IgG4-RD is extremely rare. Herein, we report a case of IgG4-RD presenting with pain in breasts, parotid masses and bilateral exophthalmos. A 52-year-old woman came to our breast disease clinic for treatment, because of pain in her breasts. After examination, eliminated the possibility of breast cancer. Physical examination indicated bilateral parotid hypertrophy and a small amount of clear liquid flowed out while pressed lightly, space-occupying lesions in the upper and outer orbits of the both eyes, this condition had been existing for 6 years. The results of immunohistochemical staining indicated IgG4-positive plasma cells in parotid masses and laboratory tests revealed elevated serum IgG4 (1480 mg/dL, normal range: 3-201 mg/dL), a diagnosis of IgG4-RD of the mammary gland, parotid gland and orbit was formulated.
\end{abstract}

Keywords: IgG4-realted disease, breast pain, orbital neoplasm, parotid masses, immunohistochemistry, case report

Cite This Article: Ruixia Yang, Weijuan Song, Ting Cui, Qiuxia Ge, Li Jiang, and Lujiang Yi, "A Case Report of IgG4-related Diseases Involving Mammary Gland, Parotid Gland and Orbit." American Journal of Medical Case Reports, vol. 7, no. 4 (2019): 71-73. doi: 10.12691/ajmcr-7-4-5.

\section{Introduction}

IgG4-related disease (IgG4-RD) is a systematic diseases involving many organs. Its main character is the affected organ are infiltrated by positive IgG4 plasmocytes $[1,2,3,4]$. This disease could be alleviated by glucocorticoid and immunosuppressor. The level of serum IgG4 is even higher in patient with multi-organs involved than single organ involved, if increased by 6 to 8 times, the specificity of diagnosing this disease will be improved. Herein, we report a case of a 52-year-old woman with IgG4-RD presenting with mammary gland, parotid gland and orbit.

\section{Case Presentation}

A 52-year-old woman came to our breast disease clinic for treatment, because of pain in her breasts. After examination, no nipple discharge and erosion were found. There were lump feeling in her breasts. No enlargement was touched in the axillary lymph nodes. Related tumor markers were CA153(12.13U/mL, normal range: $<25 \mathrm{U} / \mathrm{mL}$ ), CA125(13.5 U/mL, normal range: <35 U/mL), CEA(1.91ng/mL, normal range: $<4.7 \mathrm{ng} / \mathrm{mL})$ and the method of molybdenum X-ray examination of the breast indicated no abnormality. We initially eliminated the possibility of breast cancer. The physical examination showed the patient had abnormal oral and ocular symptom, so we suspect patients coexisting with other diseases. The patient was suggested to relevant department for diagnosis and treatment. Stomatologists found out bilateral parotid hypertrophy and a small amount of clear liquid flowed out while pressed lightly. We preliminary diagnosed as "Secondary Sjogren Syndrome" (Sjogren). The results of serum autoantibodies are as follows: Antinuclear antibody (-), anti-Sjogren's syndrome A/R052 antibody (A-SSA/R052) (-), anti-Sjogren's syndrome A/R060 antibody (A-SSA/R060) (-), anti-Sjogren's syndrome B antibody (A-SSB) (-), Anti-dsDNA antibody (A-dSDNA) $(-)$, rheumatoid factors $(14.70 \mathrm{IU} / \mathrm{ml}$, normal range: $<15 \mathrm{IU} / \mathrm{ml}$ ). Ophthalmologists also found that the patient had space-occupying lesions in the upper and outer orbits of the both eyes, this condition had been existing for 6 years. Compared with the ruselts of MR3T plain scan of double eye two years ago, her MR3T plain scan result indicated "bilateral inflammatory pseudotumors, the right one unchanged and the left one had extended" (see Figure 1). To finalize the diagnosis, rheumatology experts consulted together and decided to do immunohistochemical staining with biopsy from the lump in the parotid. The result showed the patient had IgG4-positive plasma cells (see Figure 2). 
At the same time, Laboratory tests revealed elevated serum IgG4 (1480 mg/dL, normal range: 3-201 mg/dL), According the IgG4-related diseases diagnosing standard issued in 2011 [5], this patient was diagnosed with IgG4-RD involving multiple organs.
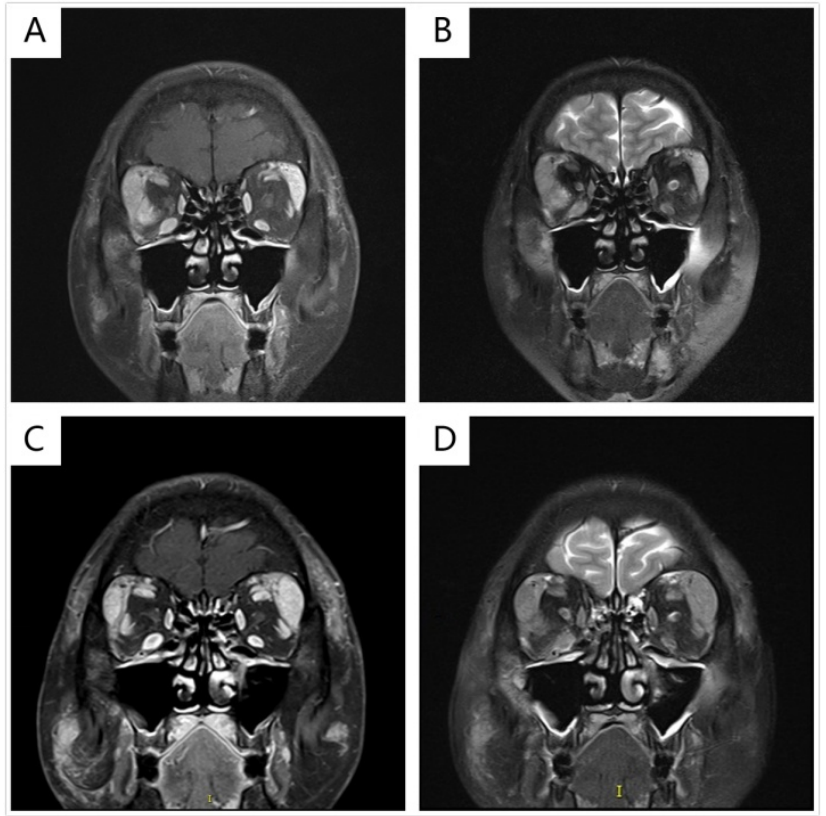

Figure 1. MR3T plain scan of double eye showing bilateral inflammatory pseudotumors, results in 2016(A) and (B), results in 2018(C) and (D)

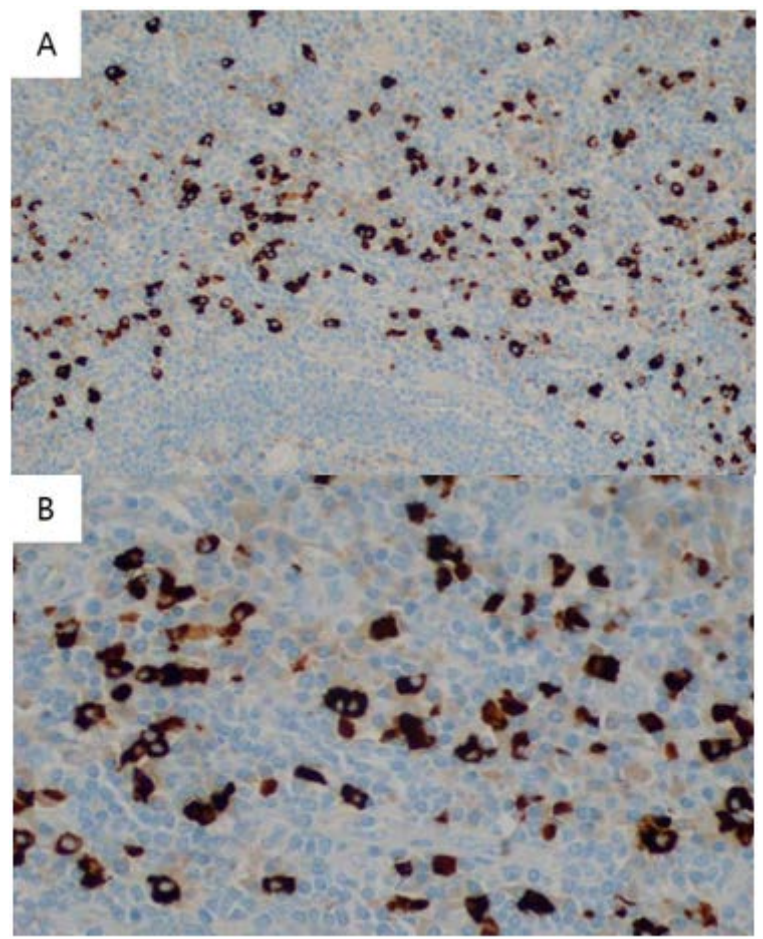

Figure 2. Immunohistochemical stains showing difffuse and intense distribution of IgG4-positive plasma cells under original magnification of 100 times (A) and 400 times (B)

\section{Discussion}

We present a rare case of IgG4-RD, which involved mammary gland, parotid gland and orbit. The study indicates that increased serum level of IgG4 concentrations and significant elevations in IgG4-positive plasma cells infiltration could be one of the options for the diagnosis of multiorgan IgG4-RD.

IgG4-RD is a recently discovered systemic condition, in which various organ mani- festations are linked by a similar histological appearance. It is is an emerging systemic condition characterized by mass-forming sclerosing lesions, elevated serum IgG4 concentrations, and extensive tissue infiltration by IgG4-positive plasma cells [6]. The concept was coined from autoimmune pancreatitis (AIP), which is now called type 1 AIP or IgG4-related pancreatitis [7]. Since 2001, when hyper-IgG4 was discovered in type 1 AIP, pathological studies have identified similar lesions in a variety of organs, eventually leading to the recognition of systemic IgG4-RD [8-11].

As in our case, histopathological confirmation of parotid masses is crucial to diagnose IgG4RD. Meanwhile, combining with negative serum tumor markers, the result of molybdenum X-ray examination of the breast ruled out the possibility of mammary cancer. Elevated serum IgG4 $>135 \mathrm{mg} / \mathrm{dL}$ was included in the diagnostic criteria for IgG4-RD [12]. Further more, the mean concentration of serum IgG4 level, among patients with multi-organ disease, was higher compared with those with single organ disease [13,14]. In addition, IgG4 positive cells $>30$ per HPF and IgG4 positive cells/IgG positive cells ratio $>50 \%$ strongly supported diagnosis of IgG4-RD [5]. According to our case, the elevated serum IgG4 level and IgG4-positive plasma cells infiltration may function as a simple suggestive role in early diagnosis.

In summary, this is a case of IgG4-RD affecting mammary gland, parotid gland and orbit. With the ruselts of MR3T plain scan of double eye and immunohistochemistry of parotid gland tissue, we could distinguish with malignancies and make a diagnosis of IgG4-RD. The data emphasize the importance of increased serum IgG4 concentrations and elevated IgG4-positive plasma cells for the diagnosis of IgG4-RD [15,16]. Early diagnosis and treatment of IgG4-RD is clinically significant because the better effectiveness of glucocorticoids in the majority of patients, could prevent irreversible organ damage.

\section{Acknowledgements}

We are grateful to the technical support from National Key Clinical Department of Laboratory Medicine of Jiangsu Province Hospital.

\section{Disclosure of Conflict of Interest}

None.

\section{References}

[1] Fong CW, Lio LI, Pon M, Mok TM, Hou N. IgG4-Related Aortitis. Eur J Case Rep Intern Med. 2018; 27; 5(9): 000881.

[2] Wallace ZS, Zhang Y, Perugino CA, Naden R, Choi HK, Stone JH; ACR/EULAR IgG4-RD Classification Criteria Committee. Clinical phenotypes of IgG4-related disease: an analysis of two 
international cross-sectional cohorts. Ann Rheum Dis. 2019; 78(3): 406-412.

[3] Slim D, Gunawardena H, Calvert JM, Daly RS, Plummeridge MJ, Medford AR. IgG4-related pulmonary disease: the protean impersonator? J R Coll Physicians Edinb. 2018; 48(2): 130-133.

[4] Fernandes B, Anacleto R, Carvalho L. IGG4 Disease And Slerosing Aortitis. Rev Port Cir Cardiotorac Vasc. 2017;24(3-4): 162.

[5] Umehara H, Okazaki K, Masaki Y, Kawano M, Yamamoto M, Saeki T, Matsui S, Yoshino T, Nakamura S, Kawa S, Hamano H, Kamisawa T, Shimosegawa T, Shimatsu A, Nakamura S, Ito T, Notohara K, Sumida T, Tanaka Y, Mimori T, Chiba T, Mishima $\mathrm{M}$, Hibi $\mathrm{T}$, Tsubouchi $\mathrm{H}$, Inui $\mathrm{K}$ and Ohara $\mathrm{H}$. Comprehensive diagnostic criteria for IgG4-related disease (IgG4-RD), 2011. Mod Rheumatol 2012; 22: 21-30.

[6] Inoue D, Yoshida K, Yoneda $\mathrm{N}$, Ozaki $\mathrm{K}$, Matsubara $\mathrm{T}$, Nagai K, Okumura K, Toshima F, Toyama J, Minami T, Matsui O, Gabata T, Zen Y. IgG4-related disease: dataset of 235 consecutive patients. Medicine (Baltimore). 2015; 94(15): e680.

[7] Hamano H, Kawa S, Horiuchi A, Unno H, Furuya N, Akamatsu T, Fukushima M, Nikaido T, Nakayama K, Usuda N, Kiyosawa K. High serum IgG4 concentrations in patients with sclerosing pancreatitis. N Engl J Med. 2001 8; 344(10):732-8.

[8] Chen JH, Deshpande V. IgG4-related Disease and the Liver. Gastroenterol Clin North Am. 2017 Jun;46(2):195-216.
[9] Meridor K, Levy Y. Immunoglobulin G4-Related Disease Presenting with Clinical Similarity to Churg-Strauss Syndrome. Isr Med Assoc J. 2019; 21(2): 124-125.

[10] Kadowaki H, Amiya E, Hoshino Y, Tamura M, Uehara M, Nakayama A, Nawata K, Domoto Y, Saeki T, Ono M, Komuro I. Enormous Aneurysm in Coronary Artery Fistula With Immunoglobulin G4-Related Disease. Can J Cardiol. 2019; 35(2): 230. e1-230. e3.

[11] Zhou Y, Shao L, Ruan W, Jin J, Xu H, Ying K, Wu X. Pulmonary vascular involvement of IgG4-related disease: Case series with a PRISMA-compliant systemic review. Medicine (Baltimore). 2019; 98(6): e14437.

[12] Tabata T, Kamisawa T, Takuma K, Egawa N, Setoguchi K, Tsuruta K, Obayashi T and Sasaki T. Serial changes of elevated serum IgG4 levels in IgG4-related systemic disease. Intern Med 2011; 50: 69-75.

[13] Kawa S, Skold M, Ramsden DB, Parker A, Harding SJ. Serum IgG4 Concentration in IgG4-Related Disease. Clin Lab. 2017 Sep 1; 63(9): 1323-1337.

[14] Carruthers MN, Khosroshahi A, Augustin T, Deshpande V and Stone JH. The diagnostic utility of serum IgG4 concentrations in IgG4related disease. Ann Rheum Dis 2015; 74: 1418.

[15] Rzepecka A, Babińska A, Sworczak K. IgG4-related disease in endocrine practice. Arch Med Sci. 2019 Jan; 15(1): 55-64.

[16] Mimori T. IgG4-related disease - A new disease entity developed in the twenty-first century. Mod Rheumatol. 2019; 29(2): 213.

(C) The Author(s) 2019. This article is an open access article distributed under the terms and conditions of the Creative Commons Attribution (CC BY) license (http://creativecommons.org/licenses/by/4.0/). 